\title{
REVUE
}

\section{LA CORROSION DES MÉTAUX AU COURS DE LA FABRICATION DU LAIT CONDENSÉ}

\author{
par \\ G. GÉNIN
}

Ingénieur E. P. C. (Paris).

De nombreuses études ont été publiées concernant la corrosion des métaux par le lait, au cours des transformations successives que ce produit subit dans l'industrie laitière. Certains chercheurs $[1,2,3$, $4,5,22]$ ont étudié l'influence des métaux sur le lait et ont constaté que le lait ou les produits qui en dérivent sont sensibles, d'une façon ou d'une autre, à l'action de certains métaux. D'autres spécialistes $[6,7,8,9,10,11,12,22]$ ont étudié, au contraire, l'influence du lait sur les métaux et ont constaté que certains métaux résistent beaucoup mieux à l'action du lait que d'autres. Nous citerons également les recherches de quelques chimistes $[13,14]$ qui ont étudié l'action, sur les métaux, de certains liquides employés dans les industries laitières, comme, par exemple, les solutions servant au nettoyage des récipients, à leur stérilisation, ou les saumures. Enfin, pour terminer, nous rappellerons quelques études [15 et 16] concernant les propriétés mécaniques des métaux en vue de leur application pratique dans l'industrie laitière. Une étude bien conduite du choix d'un métal employé dans la construction des appareils pour l'industrie laitière doit tenir compte de tous ces facteurs

Plus récemment, divers auteurs américains, appartenant soit à une compagnie laitière, soit à une compagnie métallurgique, spécialisée dans le traitement du nickel [17], se sont plus spécialement attachés à l'étude de la corrosion des métaux dans la fabrication du lait condensé et du lait condensé sucré, ce point n'ayant pas été étudié d'une façon approfondie jusqu'ici. L'expérience a montré qu'à ce point du traitement du lait, la corrosion peut être plus importante que partout ailleurs et que, dans ce cas particulier, certains métaux résistent beaucoup mieux que d'autres. Nous avons reproduit dans le tableau ci-dessous la composition des différents métaux ou alliages qui ont été étudiés par ces auteurs. Nos lecteurs retrouveront dans ce tableau des métaux qui sont très courants dans l'industrie laitière, sauf un, l'inconel, dont l'apparition est relativement récente et qui, au moment où ces essais ont été effectués, ne se trouvait pas encore dans le commerce. C'est la raison pour laquelle les études entreprises sur cet alliage n'ont porté que sur sa 
puis à l'alcool et à l'éther, séchés dans l'air, et pesés dans une balance sensible aux deux dixièmes de milligramme. Après chaque essai, les éprouvettes étaient soigneusement lavées, en utilisant uniquement de l'eau savonneuse, puis rincées, séchées et pesées comme précédemment. Dans le but de simplifier les résultats numériques obtenus, il a été décidé d'adopter une unité de perte de poids calculée en milligramme par décimètre earré de surface métallique immergée et par journée de 24 heures.

Enfin, les échantillons étaient soumis à des examens répétés, ayant pour objet de déceler la présence de corrosion locale, de piqûres ou l'apparition de taches dues à la corrosion.

Suivant que le lait est destiné à la fabrication du lait condensé sucré, ou à la fabrication du lait condensé, il subit un certain nombre de manipulations et de traitements, qu'il importe de décrire rapidement, afin de justifier les emplacements qui furent choisis pour étudier la corrosion des métaux par le lait.

Dans la fabrication du lait condensé sucré, le lait reçu dans l'atelier, est tout d'abord pesé, puis dirigé au moyen d'une pompe dans un réservoir, et enfin, après avoir passé dans un réfrigérant, il est recueilli dans des réservoirs destinés à l'emmagasiner. De là, il est dirigé dans un nouveau réservoir taré, puis dans un récipient pouvant être chauffé, où on l'additionne de la quantité voulue de sucre granulé. Dans ce dernier récipient, le sucre se dissout dans le lait; le mélange est alors porté à la température appropriée, et dirigé dans l'évaporateur à vide. Lorsque la concentration est terminée, le lait sucré et concentré est versé dans un réeipient situé au-dessous de l'évaporateur, puis, par l'intermédiaire d'une pompe, il est dirigé dans un réfrigérant, et finalement recueilli dans des réservoirsmagasins qui alimentent les machines automatiques servant au remplissage des boîtes de lait condensé.

Dans la fabrication du lait eondensé, le lait reçu dans l'atelier est pesé et dirigé vers des réservoirs où on le stocke en vue de son utilisation prochaine. Parfois, il est avant son évaporation et son stockage, refroidi, mais cette opération n'est pas nécessaire. A la sortie des réservoirs de stockage, le lait passe dans des réchauffeurs, puis dans un récipient chauffé avant d'être dirigé dans l'évaporateur travaillant sous le vide. Ce dernier opère d'une façon continue; aussi, est-il alimenté continuellement en lait, qui se déverse à la sortie de l'appareil dans un récipient situé au-dessous de l'évaporateur, puis dans un appareil destiné à homogénéiser le lait, c'est-à-dire à rompre les globules de matière grasse. Le lait homogénéisé passe ensuite dans un réfrigérant, et il est finalement recueilli dans les réservoirs servant à l'alimentation des machines de remplissage des boîtes. Ces dernières passent dans un stérilisa- 
teur ; elles sont ensuite refroidies et étiquetées et conditionnées.

Pour étudier l'action du lait sur les différents métaux, on a placé les éprouvettes métalliques à différents endroits de l'atelier de fabrication du lait condensé, ou du lait condensé sucré. En règle générale, les éprouvettes étaient placées dans le circuit du lait avant le début de la fabrication, et elles étaient retirées après une opération correspondant à une journée de travail. Après nettoyage de ces éprouvettes et pesée, elles étaient replacées dans les appareils, de telle sorte que les échantillons subissent au moins deux journées d'attaque et qu'en chaque point deux échantillons d'un même métal aient été suceessivement examinés.

Examinons maintenant les résultats de ces différents essais en tenant compte des emplacements servant à la localisation des éprouvettes.

Evaporateur. - Les éprouvettes ont été placées à trois endroits différents des appareils servant à la concentration du lait, constitués par de grands récipients en cuivre rouge. Certaines étaient fixées à la partie inférieure de l'évaporateur, de telle sorte qu'elles étaient toujours immergées dans le liquide; d'autres, au contraire, étaient placées à la partie supérieure des appareils, de telle sorte qu'elles étaient soumises à l'action de la vapeur d'eau entraînant avec elles une certaine quantité de lait sous la forme d'un fin brouillard. Enfin, la troisième série d'éprouvettes était placée à la surface du liquide soumis à l'ébullition. Les évaporateurs fonctionnaient d'une façon continue pendant 6 heures chaque jour à la température de $65^{\circ}$ sous un vide de $690 \mathrm{~mm}$. de mercure. Le lait réchauffé était introduit d'une façon continue dans l'appareil à la vitesse de $5.000 \mathrm{~kg}$. à l'heure et le lait concentré était évacué et recueilli dans un récipient d'évacuation à la cadence de $2.250 \mathrm{~kg}$. à l'heure.

Les mêmes essais furent également effectués sur un évaporateur, traitant du lait préalablement sucré. La durée de fonctionnement de cet appareil était de 7 heures par jour, et au lieu d'être alimenté d'une façon continue, il recevait des charges successives de lait contenant une certaine quantité de sucre en solution; la concentration s'effectuait pendant le temps suffisant pour arriver au produit voulu. A ce moment, l'évaporateur était vidé par simple rupture du vide et le lait condensé recueilli dans le récipient d'évacuation. Chaque opération, portuant initialement sur $2.250 \mathrm{~kg}$. de lait contenant $395 \mathrm{~kg}$. de sucre dissous, permettait d'obtenir par heure $860 \mathrm{~kg}$. de lait condensé sucré. La température du liquide dans l'évaporateur était maintenue à $60^{\circ}$ et le vide équivalent à $690 \mathrm{~mm}$. de mercure.

On a constaté au cours de ces essais que la corrosion est géné- 
ralement plus importante au eours de l'évaporation du lait normal que du lait sucré. Trois facteurs sont cause de cette différence : l'appareil traitant le lait normal travaille d'une façon continue et reçoit, de ce fait, une plus grande quantité de liquide, ce qui active la corrosion. Cette alimentation continuelle de liquide a pour conséquence également de maintenir la concentration élevée en oxygène dans le liquide, car le lait qui pénètre dans l'appareil contient une certaine proportion d'air en solution. Enfin, le liquide fourni par le lait normal présente une viscosité plus faible que les laits condensés sucrés et sa température est légèrement supérieure, facteurs qui activent la corrosion des métaux.

On a également constaté au cours de ces essais que le euivre est un des métaux les plus susceptibles à la corrosion, et pourtant, c'est ce métal qui est choisi pour la construction des évaporateurs. On a constaté avec ce métal une corrosion maximum correspondant à un indice de 73 , e'est-à-dire à une perte de $73 \mathrm{mgr}$. par décimètre carré de métal et par jour de 24 heures. Si on considère un appareil travaillant normalement 8 heures par jour pendant la moitié d'une année, on constate que la corrosion correspond à une pénétration du lait dans le métal d'environ $0 \mathrm{~mm}$. 05. Comme malgré cela, il n'a jamais été signalé de rupture d'évaporateur en cuivre servant à la concentration du lait, on peut en déduire que le remplacement de ce métal par n'importe quel autre doit donner d'excellents résultats.

En se servant de ees chiffres, on a également pu estimer la quantité de euivre passant dans le lait par suite de la corrosion du métal. Cette quantité correspond à 1,6 partie de cuivre par million de parties de lait concentré ordinaire, et 2,5 parties de cuivre dans le cas du lait condensé sucré. Ces chiffres sont d'ailleurs en accord assez étroit avee les résultats des analyses effectuées par RICE et Miscali [18], qui ont trouvé dans 16 échantillons de lait condensé sucré et dans 6 échantillons de lait eondensé contenu dans des boîtes de fer-blanc une quantité de cuivre variant de 2,4 à 4,8 parties par million de parties de lait. Si on retranche en effet de ces résultats la proportion de cuivre contenu normalement dans le lait naturel (cette quantité varie de 0,1 à 1,3 p. p. m.) ainsi que la quantité de euivre qui peut provenir de l'attaque des autres parties de l'appareillage par le lait, on trouve des résultats tout à fait comparables à ceux qui découlent de l'examen de la corrosion des évaporateurs.

Récipient d'évacuation. - Des éprouvettes des différents métaux ont été également placées à la partie inférieure des récipients d'évaeuation dans lesquels on recueille le lait à sa sortie des évaporateurs. Dans ces appareils, le lait est à la température d'environ 60 à $65^{\circ}$, il est exposé à l'air et il est dans un état continuel de monvement. On a constaté dans ce eas que les métaux les plus 
attaqués sont le nickel et le métal Monel. Il est probable que ce résultat est dû à l'action combinée de l'air et du lait, car différents auteurs [19 et 20] ont montré que l'aération augmente sensiblement la corrosion du nickel ou du métal Monel par le lait.

Les résultats sont d'ailleurs identiques, que l'on se place dans le cas du lait naturel ou dans le cas du lait condensé sucré, avec cette différence que dans ce dernier cas, la circulation du liquide n'est pas continue et que sa température est comprise entre 55 et $60^{\circ}$.

Récipients de stockage du lait condensé et du lait condensé sucré. Les éprouvettes métalliques ont été placées à la partie inférieure de ces récipients et soumises à l'action du lait condensé pendant des durées continues de 24 à 27 heures. Dans ces appareils, le lait est maintenu à une température voisine de $10^{\circ}$, et son déplacement a pour conséquence une légère aération du liquide. On a constaté que dans ces récipients l'action du lait condensé sur les métaux est relativement peu importante. Dans le cas particulier du lait condensé sucré, la corrosion est très faible, par suite probablement de la grande viscosité de ce lait, qui ralentit la dissolution de l'air dans son sein. La température du liquide est en outre très faible, et il en résulte une diminution sensible de la vitesse de corrosion.

Réservoirs d'alimentation des évaporateurs. - Ces récipients contiennent le lait porté à une température élevée dans des réchauffeurs et alimentent d'une façon continue les évaporateurs. Dans ces appareils, le liquide est maintenu à une température voisine de $92^{\circ}$ C. et il circule d'une façon continuelle depuis les réchauffeurs jusqu'à l'évaporateur. Ces appareils sont donc en fonctionnement pendant 6 heures par jour dans le cas de la fabrication du lait condensé.

Dans ce cas de la fabrication du lait condensé sucré, ces réservoirs reçoivent du lait froid et du sucre raffiné, dans la proportion de $395 \mathrm{~kg}$. de sucre pour $2.250 \mathrm{~kg}$. de lait. Le mélange est alors porté à $88^{\circ}$, en vue d'obtenir la disisolution complète du sucre et la destruction des bactéries, moisissures, etc. Finalement, la charge du récipient est versée dans l'évaporateur et l'appareil fonctionne en moyenne 7 heures par jour.

On a constaté que la corrosion des métaux, et plus particulièrement celle du métal Monel ou du nickel, est plutôt inférieure dans les appareils travaillant en continu à ce qu'elle est dans les apparei's travaillant par intermittence. Il ne semble pas que la différence soit due à la présence de sucre dans l'un des cas ; elle serait plutôt due aux variations de température dans le cas de la fabrication discontinue et à la formation d'une pellicule protectrice dans le cas du travail en continu.

Réchauffeurs. - Les éprouvettes métalliques ont été placées 
dans la tuyauterie servant au transport du lait depuis le réchauffear jusqu'au récipient servant à l'alimentation de l'évaporateur. A cet effet, un emplacement avait été prévu dans cette tuyauterie, d'un diamètre de $125 \mathrm{~mm}$., relié à ses deux extrémités à la tuyauterie normale. Dans le cas de la fabrication du lait condensé, il passait dans cette chambre d'essai $5.000 \mathrm{~kg}$. par heure de lait chaud maintenu à $93^{\circ} \mathrm{C}$., la circulation étant sensiblement constante pendant six heures par jour.

Au cours de l'examen que l'on fit des éprouvettes à chaque fin de journée, on s'aperçut qu'elles étaient recouvertes d'une pellicule très fortement adhérente, impossible à éliminer par un simple lavage et nécessitant pour son enlèvement une immersion rapide de l'éprouvette métallique dans l'acide sulfurique à $66^{\circ}$. Bé, suivie d'un lavage dans l'eau courante. Il est probable que la formation de cette pellicule est semblable à celle qu'ont signalée TrEBLER, WESLEY et LAQUE [21] dans d'autres types de réchauffeurs. On constate d'ailleurs qu'une fois la formation de eette pellicule terminée, elle constitue un excellent film protecteur contre la corrosion, exception faite des métaux tels que le cuivre ou alliages riches en cuivre.

Réservoirs de lait froid. - Le lait, lorsqu'il arrive à l'usine de concentration, est placé dans de grands réservoirs où il est maintenu à une température de $16^{\circ} \mathrm{C}$. L'arrivée continuelle du lait maintient le liquide en un état d'agitation continue, et de ce fait, le lait se trouve exposé à l'action de l'air. Cette agitation est d'ailleurs voulue et elle est même accélérée par l'action d'un agitateur, et son but est d'empêcher la séparation de la crème. On a examiné l'attaque des métaux par le lait contenu dans ces réservoirs, et on a constaté une attaque relativement peu importante, due principalement à ce que la température du liquide est peu élevée.

De tous les essais qui précèdent, rares sont ceux qui ont permis d'observer des modifications de la coloration des métaux et ce n'est que dans les évaporateurs que l'on a pu constater une coloration des éprouvettes de cuivre, coloration qui d'ailleurs n'est pas très importante et qui est maximum pour les échantillons placés à la partie supérieure de l'évaporateur, done les échantillons au contact de la vapeur d'eau et des vésicules de lait entraîné.

On a également constaté dans des cas moins fréquents des changements de coloration des éprouvettes de cuivre, de métal Monel et de nickel, placées dans des récipients contenant le lait $\dot{a}$ une certaine température. L'alliage de fer et de ehrome contenant $17 \%$ de ce dernier métal acquiert également une coloration bleuâtre dans le récipient où se fait la dissolution du suere dans le lait. Le lait froid initial entraîne également un changement de coloration très peu important du cuivre, du métal Monel et du nickel. Enfin, le 
lait condensé froid, mais non le lait condensé sucré, peut entraîner une légère modification de la coloration de certains métaux.

Les essais qui précèdent ont été effectués en plaçant les éprouvettes métalliques au contact du lait, pendant une ou quelques journées de travail seulement. D'autres essais ont été effectués en abandonnant les éprouvettes dans les évaporateurs pendant 40 à 60 jours et en pesant les échantillons avant et après l'essai. On prenait soin chaque jour de brosser et de laver les échantillons de métaux, afin de les débarrasser de la pellicuile qui aurait pu les recouvrir.

Ces essais de longue durée ont eu pour principal objet d'étudier l'action de la mise en forme, de la soudure et de la brasure sur la corrosion des métaux, ainsi que l'influence des contacts d'autres métaux. Ces essais n'ont pas donné de résultats bien probants, ear toutes les parties métalliques et en particulier les parties soudées, se recouvrent d'une pellicule protectrice qui ralentit et même empêche la corrosion, et cette pellicule adhère très fortement sur les métaux et ne peut être éliminée par un simple lavage. Il faut, pour les détacher du métal, brosser ce dernier énergiquement, puis faire agir soit de l'acide sulfurique, soit des abrasifs très efficaces, comme la toile émeri. Si cette précaution est prise, on constate alors que la corrosion des différents métaux est sensiblement la même, sauf celle du cuivre, qui ne semble pas être protégée par l'action de la pellicule protectrice.

En conclusion de tous ces essais, on peut dire que la corrosion est surtout importante dans les parties de l'appareil où se trouve le lait porté à une certaine température. Dans la majorité des cas, la résistance à la corrosion du cuivre est nettement surpassée par celle des autres métaux de bonne qualité et la supériorité de ces derniers métaux est encore plus sensible dans les points où la corrosion est la plus importante. Autrement dit, presque tous les métaux constituent une matière première supérieure au cuivre pour la construetion des évaporateurs. Pratiquement, la protection des métaux se trouve réalisée automatiquement par la formation d'une pellicule protectrice formée aux dépens du lait, pellicule protectrice dont l'action est cependant moins efficace dans le cas du cuivre.

\section{BIBLIOGRAPHIE}

[1] E. S. Gurhrie, C. L. Roadhouse et G. A. Richardson. Hildardia, 1931, t. V, p. 425 .

[2] A. F. Hess et M. J. Weinstook. Am. Med. Assoc., 1924, t. LXXXII, p. 952.

[3] O. F. Hunziker, W. A. Cordes et B. H. Nissen. J. Dairy Sci., 1929, t. XII, p. 140.

[4] A. D. Pratr. J. Nutrition, 1930, t. III, p. 141.

[5] F. E. Rice. J. Dairy Sci, 1926, t. IX, p. 459. 
[6] H. T. Gebhardt et H. H. Sommer. J. Dairy Sci, 1931, t. XIV, p. 416.

[7] H. T. Gebhardt et H. H. Sommer. J. Dairy Sci., 1932, t. XV, p. 42.

[8] O. F. Hunziker, W. A. Cordes et B. H. Nissen. Loc. cit.

[9] R. J. MoKay, O. B. Fraser et H. E. Searle. Am. Inst. Mining Met. Eng., Tech. Bull. 192, 1929.

[10] J. Miscall, G. W. Cavanaugh et P. P. Caredemos. J. Dairy Sci., 1929, t. XII, p. 379.

[11] G. N. QUaM. Ind. Eng. Chem., 1929, t. XXI, p. 703.

[12] H. A. Trebler, W. A. Wesley et F. L. Laque. Ind. Eng. Chem., 1932, t. XXIV, p. 339.

[13] L. W. Hostettler. Pa. Assoc. Dairy and Milk Insp. 8th report, 1932, p. 126.

[14] Hunziker et collaborateurs. Loc, cit.

[15] F. L. Laque. Intern. Assoc. Ice Cream Mfrs., Rept. Proc. 32 nd convention, 1932 , t. II, p. 66.

[16] H. E. SeARLE. Milk Plant Monthly, 1930, nº 3, t. XIX, p. 17.

[17] E. C. Thompson, R. P. Mears, H. E. Searle et F. L. Laque. Ind. Eng. Chem., 1934, t. XXV, p. 1311.

[18] R. J. MoKay, O. B. J. Fraser et H. E. Searte, Loc. cit.

[18 bis] F. E. Rice et J. Miscalu. J. Dairy Sci., 1923, t. VI, p. 261.

[19] MoKAY et ses collaborateurs. Loc. cit.

[20] Trebler et ses collaborateurs. Loc. cit.

[21] Ibid.

[22] C. G. Fink et F. A. Rohrman. J. of Dairy Sci., 1932, V, xv, p. 73. Le Lait, $n^{\circ} 140$, p. $1060 ; n^{\circ} 141$, p. 28.

\section{BIBLIOGRAPHIE ANALYTIQUE}

\section{LES LIVRES}

\section{Bonadonna (T.). - Basi teorichee pratiche dell'organizzazione} tecnica delle società degli allevatori di bovini da latte (Bases théoriques et pratiques de l'organisation technique de la Société des éleveurs de bétail laitier). Préface du $\mathrm{D}^{\mathrm{r}}$ Mario Muzzarini. 1 vol. de 438 pages (in-octavo). Tabl. Nombreuses photos. - Edité à Pavie, par « Ticinum ». Prix : 35 lires, ou 30 lires par l'intermédiaire de la Société d'élevage, ou demandé directement à l'auteur.

Nous ne saurions mieux présenter cet important ouvrage qu'en nous reportant à la préface signée du $D^{r}$ Mario Muzzarin.

Le sujet très vaste a été divisé en 6 parties : la première partie traite dés bases de l'extension de l'organisation zootechnique et passe en revue les buts fondamentaux, les principes substantiels, en précisant la situation italienne et étrangère.

La seconde partie développe les bases techniques des prémisses anatomophysiologiques, à la théorie de l'hérédité biologique, à la conception moderne de la sélection, à l'évaluation zootechnique des reproducteurs.

La troisième partie traite de l'organisation technique. Elle discute de l'orga. 